Urologe 2011 · 50:672

DOI 10.1007/s00120-011-2573-z

Online publiziert: 29. April 2011

(c) Springer-Verlag 2011

\author{
D. Schultheiss ${ }^{1} \cdot$ F.H. Moll ${ }^{2}$ \\ ${ }^{1}$ Urologische Belegabteilung, Evangelisches Krankenhaus Mittelhessen \\ und Gemeinschaftspraxis für Dermatologie und Urologie, Gießen \\ ${ }^{2}$ Krankenhaus Holweide, Köln
}

\title{
Der Mann hinter dem Preis
}

\section{Wissenschaftspreise - sinnvolles Steuerungs- instrument oder "Jahrmarkt der Eitelkeiten"?}

Wissenschaftspreise haben sich seit langem zu einem Steuerelement und Motivationsinstrument in der Forschungslandschaft entwickelt und dienen so der Förderung von Wissenschaft und Forschung auf vielen Ebenen. Sie sind zudem Anerkennung der Deutschen Gesellschaft für Urologie oder der urologischen Regionalgesellschaften für besondere Leistungen in ganz unterschiedlichen Bereichen des Fachgebiets. Leider sind die ausgelobten Preise im Bereich der Urologie gerade auf nationaler Ebene sowie deren Hintergründe und die Preisintention in der Fachöffentlichkeit vielfach unbekannt geblieben.

Während bis in die 1990er Jahre nur der 1928 von Alexander von Lichtenberg (1880-1949) gestiftete Maximilian-NitzePreis als höchste wissenschaftliche Auszeichnung für herausragende wissenschaftliche Leistungen bestand [1], entwickelte sich ab den 1990er Jahren des letzten Jahrhunderts im Rahmen der Modernisierung und Zukunftsausrichtung unserer Fachgesellschaft eine breite Förderung vielfältiger Gebiete.

Daher hat es sich der Arbeitskreis Geschichte der Urologie zur Aufgabe gemacht, die ausgelobten Preise der DGU, deren Intentionen und Förderungszweck sowie die Namensgeber der Preise näher $\mathrm{zu}$ beleuchten, besonders die in Verbindung mit diesen Preisen stehenden vielfältigen Leistungen dieser historischen Persönlichkeiten der Urologie darzustellen und so den „Mann hinter dem Preis“ näher zu charakterisieren und einer breiten Öffentlichkeit vorzustellen. Hierbei soll nicht einer verstaubten, heute obsoleten Hagiographie oder naiven Fortschrittsgeschichtsschreibung gehuldigt werden, sondern die wissenschaftlichen Leistungen der Namensgeber auch in das historische und wissenschaftliche Umfeld der jeweiligen Epoche in Bezug gesetzt werden. Gleichzeitig stehen die heutigen Preisträger in Beziehung zu aktuellen und innovativen Forschungsansätzen oder verkörpern arrivierte ausgewiesene Persönlichkeiten des Fachgebiets.

In der Rubrik „Geschichte der Urologie“ wird in dieser Ausgabe als erster Beitrag der Reihe das Leben und Werk Anton Ritter von Frischs (1849-1917) vorgestellt. Weitere Namensgeber von DGU-Preisen werden in nächster Zeit vorgestellt.

Gerade das weite Spektrum der Preisintentionen verdeutlicht den interdisziplinären Ansatz der Urologie sowie die enge Verbindung zwischen Klinik und Praxis im Fachgebiet. Während der Kieler Medizinhistoriker Robert Herrlinger (19141968) in den 196oer Jahren in Bezug auf den Nobelpreis „von einem Jahrmarkt von Eitelkeiten“ sprach [2], so kann doch bei der Liste der Preisträger für die verschiedenen Preise innerhalb der Urologie gelten, dass sich für die ausgezeichneten Persönlichkeiten ein stets breiter Konsens finden ließ.

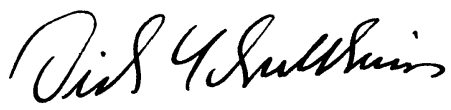

D. Schultheiss

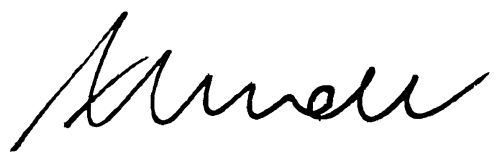

F.H. Moll

\section{Korrespondenzadresse \\ Prof. Dr. D. Schultheiss \\ Urologische Belegabteilung, \\ Evangelisches Krankenhaus Mittelhessen \\ und Gemeinschaftspraxis \\ für Dermatologie und Urologie, \\ Friedrichstraße 21, 35392 Gießen \\ dirk.schultheiss@urologie-giessen.de}

\section{Literatur}

1. Rathert P (2004) Zur Geschichte des MaximilianNitze-Preises. Vortrag anlässlich des Preisträger Essens auf dem 56 . Kongress der DGU, Wiesbaden (unveröffentlichtes Redemanuskript)

2. Der Spiegel (1967) Die Nobelpreisträger der Medizin. Der Spiegel, München, Bd 44, S 267-268 